\title{
Broca's Area Supports Enhanced Visuospatial Cognition in Orchestral Musicians
}

\author{
Vanessa Sluming, ${ }^{1,2}$ Jonathan Brooks, ${ }^{3}$ Matthew Howard, ${ }^{2,4}$ John Joseph Downes, ${ }^{5}$ and Neil Roberts ${ }^{2}$ \\ ${ }^{1}$ School of Health Sciences' Division of Medical Imaging and Radiotherapy, University of Liverpool, Liverpool L69 3GB, United Kingdom, ${ }^{2}$ Magnetic \\ Resonance and Image Analysis Research Centre, University of Liverpool, Liverpool L69 3GE, United Kingdom, ${ }^{3}$ Centre for Functional Magnetic Resonance \\ Imaging of the Brain, Department of Clinical Neurology, Oxford University, John Radcliffe Hospital, Oxford OX3 9DU, United Kingdom, ${ }^{4}$ Centre for \\ Neuroimaging Sciences, Institute of Psychiatry, London SE5 8AF, United Kingdom, and ${ }^{5}$ School of Psychology, University of Liverpool, Liverpool L69 7ZA, \\ United Kingdom
}

We provide neurobehavioral evidence supporting the transferable benefit of music training to alter brain function and enhance cognitive performance in a nonmusical visuospatial task in professional orchestral musicians. In particular, orchestral musicians' performance on a three-dimensional mental rotation (3DMR) task exhibited the behavioral profile normally only attained after significant practice, supporting the suggestion that these musicians already possessed well developed neural circuits to support 3DMR. Furthermore, functional magnetic resonance imaging revealed that only orchestral musicians showed significantly increased activation in Broca's area, in addition to the well known visuospatial network, which was activated in both musicians and nonmusicians who were matched on age, sex, and verbal intelligence. We interpret these functional neuroimaging findings to reflect preferential recruitment of Broca's area, part of the neural substrate supporting sight reading and motor-sequence organization underpinning musical performance, to subserve 3DMR in musicians. Our data, therefore, provide convergent behavioral and neurofunctional evidence supporting the suggestion that development of the sight-reading skills of musical performance alters brain circuit organization which, in turn, confers a wider cognitive benefit, in particular, to nonmusical visuospatial cognition in professional orchestral musicians.

Key words: visuospatial; cognition; human; musician; Broca's area; mental rotation

\section{Introduction}

The extensive occupational engagement of musicians who are members of a professional symphony orchestra provides an opportunity to study the potential long-term effects associated with acquiring and sustaining expert musical performance skills. In particular, the human brain has a well known capacity for neuroplastic adaptation in response to skill acquisition (Sterr et al., 1998), which is predicted to occur in musicians in structures that have particular functional relevance for enabling musical performance. For example, increased gray-matter volume in Broca's area, known to subserve the visuospatial analysis and sequencing of rapid motor actions required for musical performance from sight reading (Sergent et al., 1992; Parsons et al., 2005; Bengtsson and Ullen, 2006), was found to be related to years of orchestral playing (Sluming et al., 2002). Sight-reading ability has been shown to be a significant factor influencing a musician's ability to perform a repertoire of rehearsed music (McPherson et al., 1997), which is fundamental for professional symphony orchestra mu-

\footnotetext{
Received July 19, 2006; revised Feb. 27, 2007; accepted March 1, 2007.

We thank the radiographers at the Magnetic Resonance and Image Analysis Research Centre and Walton Centre for Neurology and Neurosurgery (Liverpool, UK) for their assistance.

Correspondence should be addressed to Vanessa Sluming, The University of Liverpool, School of Health Sciences, Thompson-Yates Building, The Quadrangle, Brownlow Hill, Liverpool L69 3GB, UK. E-mail: vanessa.sluming@liverpool.ac.uk.

DOI:10.1523/JNEUROSCI.0147-07.2007

Copyright $\odot 2007$ Society for Neuroscience $\quad$ 0270-6474/07/273799-08\$15.00/0
}

sicians. It is a complex skill that occurs automatically in the highly skilled sight reader (Wolf, 1976) and involves the extremely rapid implementation of a series of operations through perception, including visuospatial analysis of symbolic input, to the sequential and coordinated motor output of musical performance (Sergent, 1993). Furthermore, sight reading is characterized by high demands on the performer's capacity to process complex visual input under real-time constraints and with no opportunity for error correction (Kopiez et al., 2006). We therefore used the classic three-dimensional mental rotation (3DMR) task of Shepard and Metzler (1971), which could be conveniently administered to both musicians and nonmusicians to quantify visuospatial analysis and mental imagery of motor transformation ability (Kosslyn et al., 1984; Wallace and Hofelich, 1992). 3DMR is a complex high-level nonmusical spatial ability test which, according to traditional theories of mental rotation, involves rapidly sequencing a series of operations, specifically (1) stimulus identification, (2) search, (3) mental rotation, and (4) decision (Shepard and Cooper, 1982). Using functional magnetic resonance imaging (fMRI) to study 3DMR, we tested the hypothesis that orchestral musicians would, in comparison with nonmusicians, show increased activation in Broca's area, known to subserve hierarchical organization of behavior by controlling the selection and nesting of action segments (Koechlin and Jubault, 2006), in addition to the visuospatial network (Cohen et al., 1996), which would be specifically associated with enhanced performance on this test, evi- 
denced by faster rotation speeds and lower error susceptibility (Just and Carpenter, 1985). As with many cognitive measures, the 3DMR task shows an age-related decrement, that is, an increase in response times and slope, in older adults (Dror and Kosslyn, 1994; Dror et al., 2005). In a previous experiment involving orchestral musicians, however, we found an age-related enhancement on Benton Judgment of Line Orientation (JOL) (Sluming et al., 2002) rather than the anticipated age-related decline found in nonmusicians. JOL is a relatively low-level visuospatial perceptual task (Collaer and Nelson, 2002) that is known to be partially subserved by similar neural networks supporting 3DMR (Barnes et al., 2000; $\mathrm{Ng}$ et al., 2001). We, therefore, also predicted agerelated enhancement in performing $3 \mathrm{DMR}$ by orchestral musicians.

\section{Materials and Methods}

Participants. The study had Local Research Ethics Committee approval. All volunteers gave signed, informed consent and were medically screened before scanning. Ten right-handed male musicians from Royal Liverpool Philharmonic Orchestra (seven played violin, two cello, and one double bass) were recruited (mean age, 42.2 years; SD, 10.1 years). The control group, matched for age, handedness, and verbal intelligence, consisted of 10 male right-handed academic staff members of the Faculties of Medicine and Science of the University of Liverpool (mean age, 40.1 years; SD, 11.8). The orchestral musicians were a subset of the volunteers recruited to our previous study (Sluming et al., 2002).

Assessment of verbal intellectual ability. We administered the speed of sentence comprehension task from the speed and capacity of language processing test (SCOLP) (Baddeley et al., 1992) as an fMRI paradigm to assess verbal intelligence (Saxton et al., 2001) in musicians and controls, both behaviorally and neurofunctionally. This sentence comprehension paradigm consisted of a visually presented series of simple declarative sentences, delivered using back projection onto a screen visible to the subjects lying in the scanner, which the participant had to read and verify as either true or false as rapidly as possible. The sentences reflect everyday, common sense world knowledge that should be known to most people (e.g., "prime ministers are made in factories" and "prime ministers are people"). Semantic as opposed to syntactic processing has been shown to activate Broca's area to a greater extent (Chee et al., 2002), as has visually presented sentences compared with spoken sentences (Carpentier et al., 2001). To control for saccadic eye movement, motor activity of button-pressing, and decision making, a control task comprising a string search in which subjects had to identify whether an hourglass appeared within pseudosentences made by converting the SCOLP sentences into a symbolic font was administered, for example,

patOM,

Stimuli for the fMRI 3DMR experiment. Visual stimuli for the 3DMR task were delivered using back projection onto a screen visible to subjects in the scanner, and presented within a block design with alternating epochs of control and task. The 3DMR stimuli were based on the classic stimuli of Shepard and Metzler (1971) and consisted of pairs of threedimensional perspective drawings of 10 cubes arranged in chiral patterns (Fig. 1) displayed on the screen side by side simultaneously. The lefthand form (viewed by the observer) is presented with the major axis vertically oriented, whereas the right-hand form is presented at eight possible angles from vertical $\left(0^{\circ}, 45^{\circ}\right.$ to $180^{\circ}$ in $45^{\circ}$ increments, clockwise and counterclockwise). Behaviorally, the test is characterized by a positive linear association between response time and angle of rotation with subjects having to make a judgment about whether a pair of shapes are identical (with one rotated relative to the other) or nonidentical. For shapes shown at different orientations, successfully completing 3DMR requires the rapid construction of a mental image and rotation of that image such that its identity can be checked against the comparator, whereas for pairs of shapes shown at the same orientation the task is simply identity recognition. To control for saccadic eye movement, motor activity of button-pressing, and decision making a control task, which

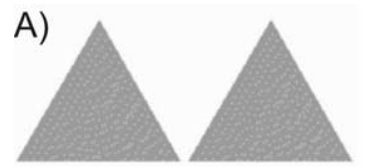

B)
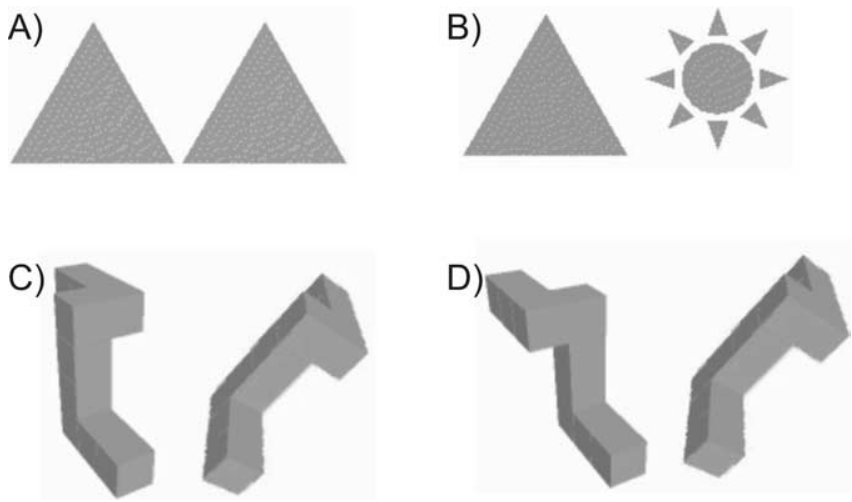

Figure 1. Examples of stimuli for the experimental tasks. $\boldsymbol{A}, \boldsymbol{B}$, Shapes for the $2 \mathrm{D}$ shape matching (control condition) in which the subjects decide whether the shapes are identical $(\boldsymbol{A})$ or not $(\boldsymbol{B})$. C, D, Pairs of congruent $(\boldsymbol{C})$ and incongruent (D) 3D perspective drawings of 10 cubes arranged in a chiral pattern with left-hand pattern of pair presented with the major axis vertical and right-hand pattern presented at one of eight angles from vertical for the 3DMR task (see Materials and Methods) in which subjects decide whether the right-hand figure was identical, but rotated, relative to left-hand figure.

required deciding whether a pair of 2D shapes (see Fig. 1) are the same or dissimilar, was administered. During the fMRI examination, the 3DMR and the $2 \mathrm{D}$ perceptual shape-matching tasks were administered in alternating epochs using a self-paced box-car design to maximize response rates and avoid down time between trials. For the $2 \mathrm{D}$ shape-matching task, participants were instructed to decide whether pairs of shapes were identical or not, whereas for the 3DMR task, the instructions were to mentally rotate the right-hand form of the pair until they could decide whether it matched, or did not match, with the left-hand form. The figures remained on the screen until the subject responded. Subjects were requested to indicate their response (match/mismatch) as quickly and accurately as possible for all tasks.

For all of the tasks administered during the verbal intellectual ability and 3DMR fMRI experiments, the subjects responded (match/correct or mismatch/incorrect) via button boxes, and response times during performance of all tasks were recorded on-line. Before entering the MR scanner, each subject was given detailed instructions and shown examples of the sentences and figures (specifically those in Fig. $1 A, B$, for 2D shape-matching, $C, D$, for $3 \mathrm{DMR})$. Subjects did not explicitly practice any of the tasks before commencement of the experiments, but were confident that they understood the requirements of each of the tasks and were instructed to respond as quickly as possible while keeping errors to a minimum.

fMRI procedure and image analysis. MR images were acquired using a 1.5T General Electric (Milwaukee, WI) LX/NVi system. Functional data were obtained using a gradient echo echo-planar imaging sequence (echo time, $40 \mathrm{~ms}$; repetition time, $3 \mathrm{~s}$; flip angle, $90^{\circ}$; $64 \times 64$ matrix; field of view, $19 \mathrm{~cm}$; slice thickness, $5 \mathrm{~mm}$ ). For each experiment (verbal intellectual ability and 3DMR), scan time was $5 \mathrm{~min}$ and consisted of 20 epochs with each epoch lasting $15 \mathrm{~s}$. Preprocessing and data analysis were performed using Statistical Parametric Mapping software (SPM99; Welcome Department of Imaging Neuroscience, London, UK) running under Matlab 6.5 (MathWorks, Natick, MA) on a Sun Sparc 10 workstation (Sun Microsystems, Santa Clara, CA). After motion correction, spatial normalization, and smoothing with a $9 \mathrm{~mm}$ full-width half-maximum kernel, grouped data (musicians and controls) were analyzed using within-groups fixed-effects maps of blood oxygen level-dependent $(B O L D)$ response signal and are reported for $p_{\text {(corrected) }}<0.05$. Withingroup analyses contrasting the $3 \mathrm{DMR}$ with the $2 \mathrm{D}$ shape-matching conditions permits identification of the neural activations associated with the mental rotation process per se (Cohen et al., 1996). Differences between activation maps for musicians and controls were assessed using a second level (random effects) analysis, which used a functional (inclusive) mask and are reported for $p_{\text {(corrected) }}<0.05$. The functional mask was created by taking the within-group fixed effect maps at $p_{\text {uncorrected }}<$ 
A

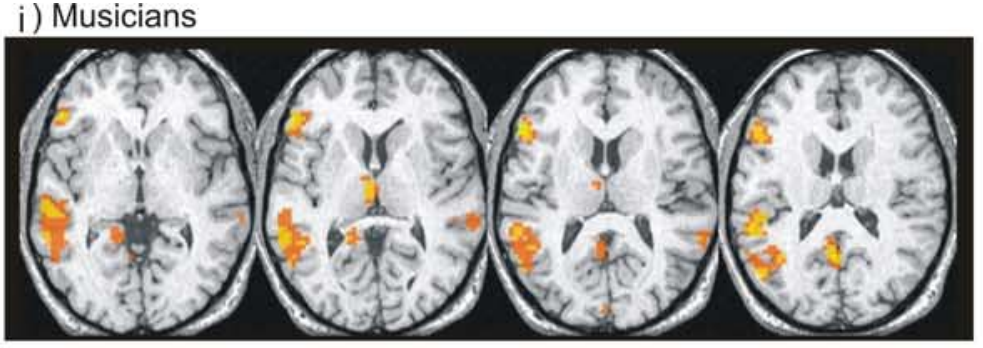

ii) Non-Musicians
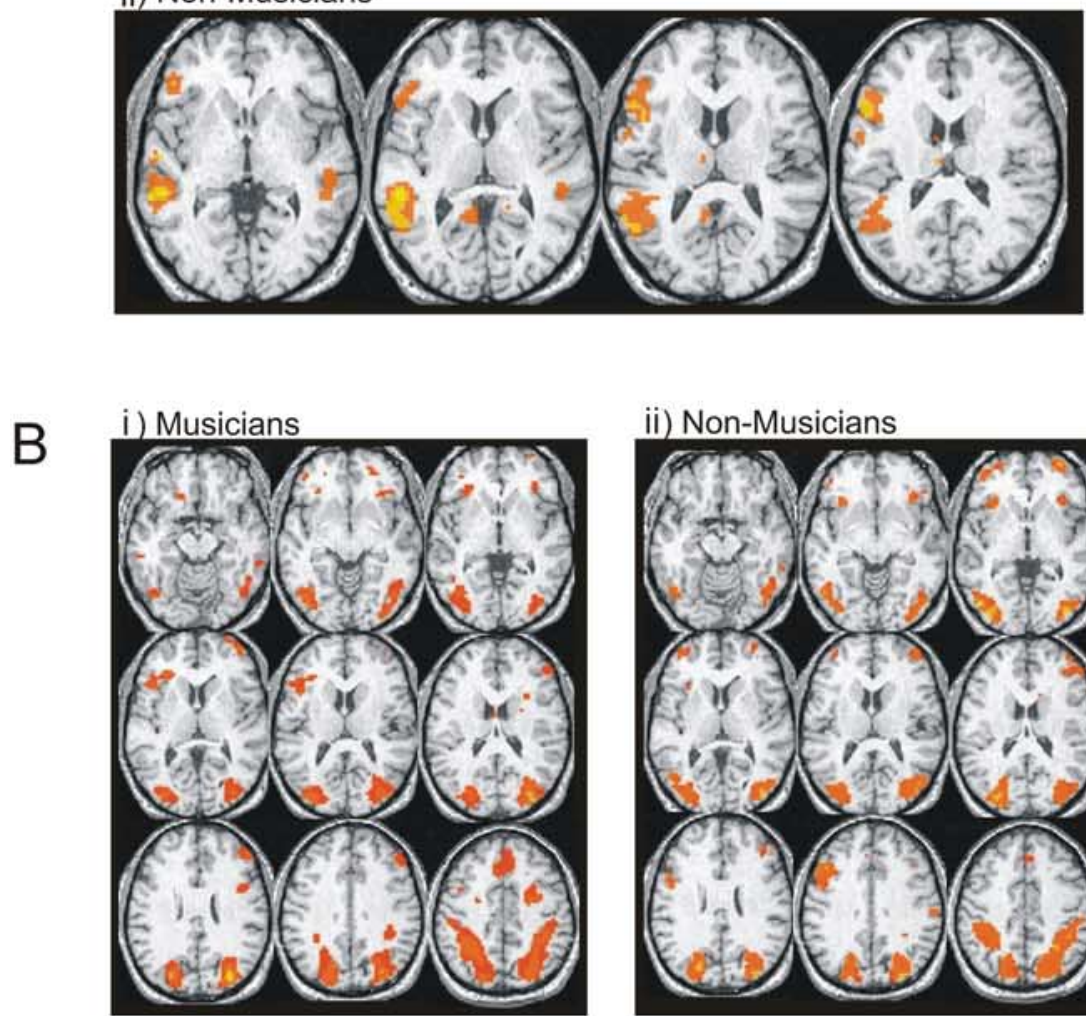

ii) Non-Musicians

iii)
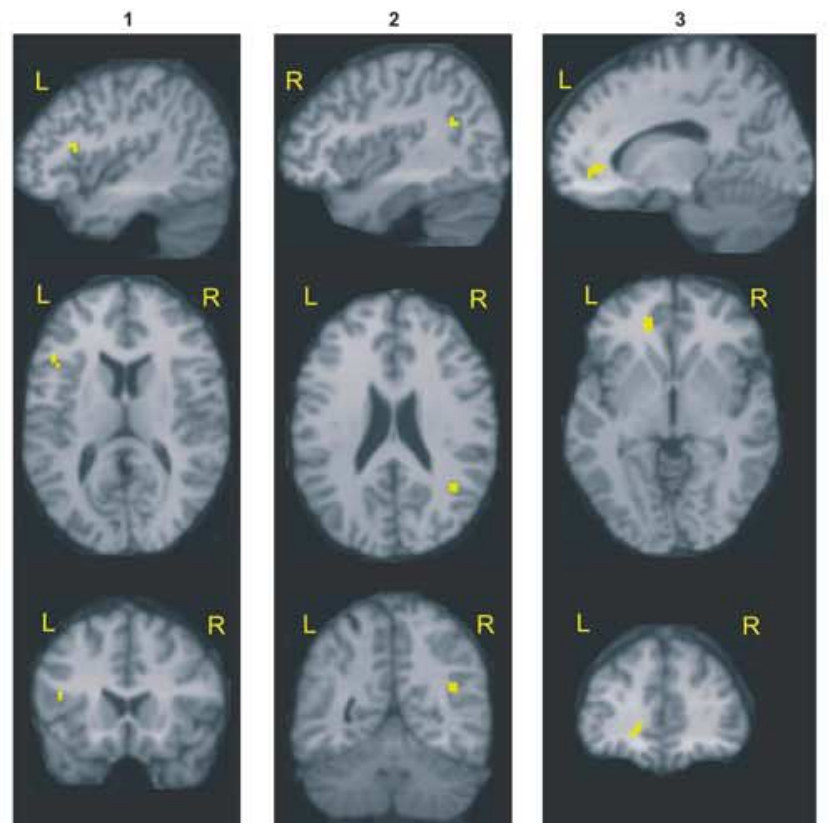

Figure 2. A, Brain activations in musicians and controls during the assessment of verbal intellectual ability. Bi, Bii, Activations resulting from within-group random-effects analyses for musicians (i) and nonmusicians (ii) at $z$-slice levels used by Sergent et al. (1992) to describe the sight-reading neural network. Top row, Broca's area in left hemisphere activated in musicians only; middle
0.001 for each group and adding them to produce a liberal functional mask, which included all areas with increased BOLD signal in both groups during performance of the 3DMR task (i.e., inclusive masking). Regions of significantly increased activation were localized using the cytoarchitectonic maximum probability maps for Brodman areas 44 and 45 (Amunts et al., 1999) provided in the Anatomy Toolbox in SPM (Eickhoff et al., 2006).

\section{Results}

Assessment of verbal intelligence

There were no differences in the total number of semantic processing $(t=0.48$; $\mathrm{df}=18 ; p=0.64)$ or control trials $(t=$ $0.37 ; \mathrm{df}=18 ; p=0.72$ ) attempted, or the total number of correct semantic processing trials $(t=-1.1 ; \mathrm{df}=18 ; p=0.30)$ or number of correct control trials $(t=0.77$; $\mathrm{df}=18 ; p=0.45$ ) between musicians and controls. Musicians had an accuracy rate of $98 \%$ in the sentence processing task and $90 \%$ in the control condition; the control group had an accuracy rate of $98 \%$ in the sentence processing task and $89 \%$ in the control condition. There was no difference in mean response times for sentence processing between the groups $(t=0.67$; $\mathrm{df}=18 ; p=0.51)$. During sentence processing, within-group random effects analysis of the fMRI data revealed that both groups activated similar cortical regions, mainly in left hemisphere, notably, the classic language regions of Broca's and Wernicke's areas (Fig. 2). There were no significant differences in brain activation patterns in the between-group random effects analysis (RFX) analysis.

\section{Behavioral results for $3 \mathrm{DMR}$ and 2D shape-matching control tasks}

The mean number (range) of trials attempted by the subjects was as follows: musicians, 2D shape-matching, 141 (116161); 3DMR, 94 (53-137); controls, 2D shape-matching, 139 (97-158); and 3DMR, 71 (45-116). Accuracy rates for the two experimental tasks were as follows: musicians, 2D shape-matching, 94\%; 3DMR, 81\%; controls, 2D shape-

\footnotetext{
$\leftarrow$

and bottom rows, premotor and parietal cortices activated in both groups. Biii, Results of the random-effects group differences analysis projected onto a structural MR scan of a subject selected at random from the volunteers, showing regions with significantly increased BOLD signal in musicians compared with nonmusicians during 3DMR task performance (column 1, left pars operculum, $x=-45, y=18, z=14$, $t=6.56,6$ voxels; column 2 , right angular gyrus, $x=42, y=$ $-54, z=23, t=4.11,6$ voxels; column 3, left anterior cingulate gyrus, $x=-15, y=38, z=6, t=5.82,14$ voxels).
} 


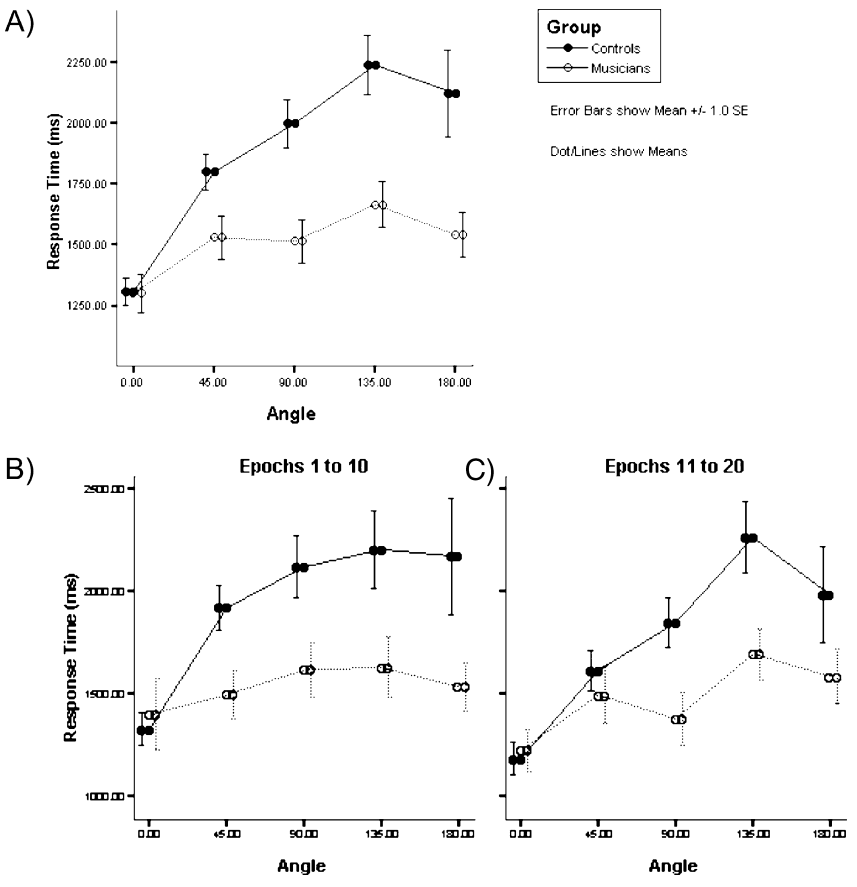

Figure 3. $A$, Mean RTs (error bars, \pm 1 SEM) as a function of angle between the paired forms of 3DMR task. The classical behavioral signature of the 3DMR task, in which RT increase linearly as a function of angle between the forms, was evident only in nonmusicians. $\boldsymbol{B}$, Mean RTs (error bars, $\pm 1 \mathrm{SEM}$ ) as a function of angle between the paired forms of the 3DMR task during epochs 1-10. C, Mean RTs (error bars, \pm 1 SEM) as a function of angle between the paired forms of the 3DMR task during epochs 11-20.

matching 95\%; and 3DMR, 73\%. There were no differences in either the number of control 2D shape-matching trials attempted $(t=0.45 ; \mathrm{df}=18 ; p=0.66)$ or scored correctly $(t=0.09 ; \mathrm{df}=18$; $p=0.93)$, indicating that both groups were performing at the same level in this control task. However, orchestral musicians attempted significantly more 3DMR trials $(t=2.8 ; \mathrm{df}=11.6 ; p=$ 0.02 ) and achieved more correct responses than the control group, both absolutely $(t=4.5$; df $=18 ; p=0.00)$ and as a proportion of trials attempted $(t=3.4 ; \mathrm{df}=18 ; p=0.003)$. The classical behavioral signature of $3 \mathrm{DMR}$, with response times (RTs) increasing linearly as a function of angle-of-rotation (Shepard and Metzler, 1971), was seen only in the control group (Fig. 3A). Analysis of median RT (correct trials, excluding outliers not within \pm 3 SD of mean for each individual) was performed using a mixed-design ANOVA, with angle and timing of epoch (epochs 1-10 categorized as "early" and epochs 11-20 as "late") as within-subject factors. Neither the main effect of epoch $\left(F_{(1,18)}=2.69 ; p=0.12\right)$ nor the interactions involving the epoch term were significant (epoch by angle by group, $F_{(1,18)}=0.31$, $p=0.86$; epoch by group, $F_{(1,18)}=0.85, p=0.37$ ) (Fig. $3 B$ ), providing evidence that performance was stable across the two halves of the fMRI scanning session. Notably, however, there was a significant group by angle interaction $\left(F_{(1,18)}=7.20 ; p=0.02\right)$ and a simple main-effects analysis identified angle of rotation as being statistically significant in nonmusicians $\left(F_{(1,9)}=16.01 ; p=\right.$ $0.003)$, but not orchestral musicians $\left(F_{(1,9)}=1.13 ; p=0.32\right)$. As can be seen in Figure $3 A$ (and for comparison across epochs, Fig. $3 B, C)$ the nonmusicians display the classic RT slope function, whereas that for the musicians was essentially flat. Simple maineffects analyses confirmed that for nonmusicians, angle of rotation was found to be significant $\left(F_{(1,9)}=16.01 ; p=0.003\right)$, whereas the effect was unreliable for orchestral musicians
$\left(F_{(1,9)}=1.13, p=0.32\right)$. The overall average slopes of these RT functions were $1.57 \mathrm{~ms} /{ }^{\circ}$ and $5.25 \mathrm{~ms} /{ }^{\circ}$ with intercepts of 1403 and $1510 \mathrm{~ms}$ for musicians and nonmusicians, respectively. Overall mean RT for 3DMR was negatively associated with age in musicians $(r=-0.68 ; p=0.03)$, whereas for nonmusicians a nonsignificant trend $(r=0.37 ; p=0.30)$ consistent with the typical age-related increase in RT was observed. Finally, for the musicians, overall mean RT was negatively correlated with number of years of orchestral playing for the 3DMR task $(r=-0.63$; $p=0.05)$, but not for the control $2 \mathrm{D}$ shape-matching task $(r=$ $-0.07 ; p=0.83)$.

\section{fMRI results for 3DMR experiment}

Within-group RFX ( $p_{\text {corrected }}=0.05$ ) of fMRI data revealed that performing $3 \mathrm{DMR}$ is associated with increased BOLD signal in similar brain regions in both musicians and nonmusicians, including bilateral visual association (BA18/19), and premotor (BA6) and superior parietal (BA7) cortices, as has been reported previously (Cohen et al., 1996) (Fig. 2 Bi,Bii, Table 1). Subsequently, RFX analysis of group differences $\left(p_{\text {corrected }}<0.05\right.$ ) revealed that there were significantly greater activations only in Broca's area (BA44 and BA45), the right angular gyrus (BA39), and left anterior cingulate cortex (ACC) (BA24/32) during 3DMR performance (Fig. 2 Biii) in musicians relative to controls. Cytoarchitectonic maximum probability maps for Brodman areas 44 and 45 (Amunts et al., 1999; Eickhoff et al., 2006) localized $62 \%$ of activation to BA 45 and $38 \%$ of activation to BA44 during 3DMR. Furthermore, the slope of the RT function for musicians was negatively correlated with activity only in Broca's area $(x=$ $-54, y=24, z=9$; $t=5.52 ; p=0.02$; two voxels, $70 \%$ probability of BA45) such that flatter slopes (i.e., better performance) were associated with increased activation in these voxels. Data at this voxel, extracted for each subject, plotted against their RT slope function is shown in Figure 4.

\section{Discussion}

We found convergent behavioral and neurofunctional evidence to support the hypothesis that orchestral musicians will show enhanced performance on the complex visuospatial task of 3DMR corresponding with increased activation in Broca's area, part of the neural network subserving sight reading. In particular, we found that musicians were, overall, more accurate on the 3DMR task and, although matched on $0^{\circ}$ trials, exhibited progressively faster RTs as a function of angular disparity. Furthermore, the averaged non $-0^{\circ} \mathrm{RT}$ s for the musicians were negatively correlated with years of orchestral experience. Neurofunctionally, in addition to the visuospatial network identified previously (Cohen et al., 1996), only musicians activated Broca's area, which was also found to be negatively correlated with their RT slope function, such that flatter slopes were associated with greater activation in this brain region. The results of the between-group random effects fMRI data analysis, therefore, suggest that increased activation of Broca's area in the musicians' group mediates faster mental manipulation of 3DMR stimuli and greater accuracy of decision making.

The prefrontal cortex, including Broca's area, is one of the last brain regions to reach maturity in the adult brain (Sowell et al., 1999), thus, retaining a high degree of neuroplasticity during adolescence and early adulthood when musicians, who progress to achieve a professional standard of performance, will be practicing their musical skills intensely (Ericsson et al., 1993). Neuroimaging studies have provided convincing evidence of differences in musicians' brains in structures known to have particular 
Table 1. Localization of significantly activated clusters identified in the within-groups RFX analysis illustrated in Figure 2, Bi and Bii.

\begin{tabular}{|c|c|c|c|c|c|}
\hline \multirow[b]{2}{*}{ Group and cluster (number of voxels) } & \multirow[b]{2}{*}{ Activated regions } & \multicolumn{3}{|c|}{ MNI coordinates } & \multirow[b]{2}{*}{ tstatistic } \\
\hline & & $x$ & $y$ & $z$ & \\
\hline \multicolumn{6}{|l|}{ Musicians } \\
\hline \multirow{4}{*}{ Cluster 1 (765) } & Left inferior parietal lobule & -36 & 65 & 53 & 12.12 \\
\hline & Left middle occipital gyrus & -27 & 82 & 32 & 11.13 \\
\hline & Left superior occipital gyrus & -24 & 88 & 35 & 9.73 \\
\hline & Left superior parietal lobule & -21 & 70 & 56 & 9.32 \\
\hline \multirow[t]{3}{*}{ Cluster 2 (731) } & Right middle occipital gyrus & 30 & -85 & 32 & 18.09 \\
\hline & Right superior parietal lobule & 24 & -49 & 59 & 12.10 \\
\hline & Right angular gyrus & 33 & -61 & 59 & 7.02 \\
\hline \multirow[t]{2}{*}{ Cluster 3 (104) } & Right middle frontal gyrus & 17 & 5 & 53 & 7.75 \\
\hline & Right superior frontal gyrus & 24 & 5 & 62 & 5.89 \\
\hline \multirow[t]{3}{*}{ Cluster 4 (35) } & Right superior medial gyrus & 6 & 29 & 50 & 6.82 \\
\hline & Left supplementary motor area & -6 & 20 & 50 & 5.08 \\
\hline & Left superior medial gyrus & -3 & 26 & 53 & 4.91 \\
\hline Cluster 5 (23) & Left inferior frontal gyrus & -45 & 14 & 20 & 6.29 \\
\hline \multirow[t]{2}{*}{ Cluster 6 (21) } & Left precentral gyrus & -27 & -7 & 65 & 5.3 \\
\hline & Left middle frontal gyrus & -24 & 5 & 65 & 5.2 \\
\hline Cluster 7 (14) & Left inferior frontal gyrus /insula & -30 & 24 & 11 & 6.56 \\
\hline \multicolumn{6}{|l|}{ Controls } \\
\hline \multirow[t]{4}{*}{ Cluster 1 (608) } & Left middle occipital gyrus & -39 & 91 & 2 & 10.56 \\
\hline & Left superior parietal gyrus & -18 & -79 & 59 & 9.91 \\
\hline & Left superior occipital lobule & -21 & 82 & 32 & 8.41 \\
\hline & Left precuneus & -6 & 70 & 62 & 8.15 \\
\hline \multirow[t]{5}{*}{ Cluster 2 (731) } & Right inferior parietal lobule & 42 & -49 & 59 & 12.83 \\
\hline & Right angular gyrus & 33 & -61 & 50 & 10.24 \\
\hline & Right superior parietal lobule & 27 & -61 & 56 & 9.39 \\
\hline & Right middle occipital gyrus & 36 & -88 & 32 & 9.12 \\
\hline & Right inferior occipital gyrus & 42 & -85 & 2 & 9.08 \\
\hline Cluster 3 (52) & Left inferior parietal lobule & -45 & -43 & 53 & 7.08 \\
\hline Cluster 4 (21) & Left inferior temporal gyrus & -61 & -70 & -1 & 8.95 \\
\hline Cluster 5 (24) & Left precentral gyrus & -48 & 5 & 38 & 6.1 \\
\hline Cluster 6 (20) & Right middle frontal gyrus & 30 & -1 & 65 & 7.04 \\
\hline Cluster 7 (12) & Supplementary motor area & 0 & 17 & 53 & 6.2 \\
\hline
\end{tabular}

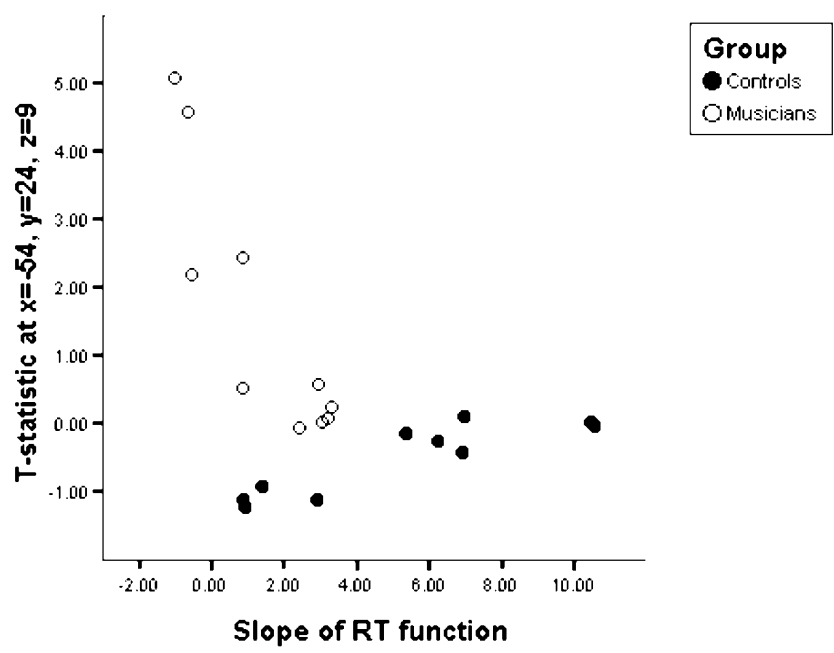

Figure 4. Scatterplot of the $t$ statistic from the first level analysis of fMRI brain data against the slope of the RT function of the behavioral data for each individual at voxel coordinates $x=$ $-54, y=20$, and $z=13$, which was identified as statistically significant $(p=0.02)$ in the correlational SPM analysis only in musicians.

relevance for enabling musical performance, such as increased cross-sectional area of the anterior corpus callosum (Schlaug et al., 1995), increased cortical representation of the left-hand fingers in string players (Elbert et al., 1995), increased gray-matter (GM) volume in anteromedial Heschl's gyrus related to pitch perception (Schneider et al., 2005), and increased GM volume in
Broca's area, related to years of playing in orchestral musicians (Sluming et al., 2002). One explanation for the findings that we report could be that inherent brain structure may predispose musicians to excel at the skills necessary for expert musical performance. Alternatively, there may be an interaction between brain development and acquisition and maintenance of musical visuospatial skills, which promotes generation or retention of functionally relevant cortical tissue, such as use-dependent adaptation in Broca's area (Sluming et al., 2002), which can be preferentially recruited to subserve complex visuospatial, nonmusical tasks such as 3DMR. This latter interpretation is supported by our findings of negative correlation between activation in Broca's area and RT in musicians and also by the neurofunctional dissociation we observed in Broca's area between verbal and visuospatial tasks.

In addition to its well known role in motor sequencing of expressive language Broca's area has been shown to subserve sequencing of nonlanguage motor functions such as preparation and performance of complex hand movements (Stephan et al., 1995; Binkofski et al., 2000), associative sensorimotor learning and integration (Binkofski and Buccino, 2004), production of actions (Bonda et al., 1995; Parsons et al., 1995), and sensory processing of visual stimuli (Mechelli et al., 2005), all of which are crucial elements of expert musical performance (Waters et al., 1997; Kopiez et al., 2006). Furthermore, it has been proposed that this region subserves hierarchical organization of behavior (Koechlin and Jubault, 2006) by implementing a specialized executive system to control the selection and nesting of action segments, with top-down control exerted from anterior (BA45) to 
posterior (BA44) regions. Sight reading is a complex process involving a reading skill of encoding musical symbols (Furneaux and Land, 1999) and a transformational sensorimotor skill of positioning the fingers appropriately on the instrument at precisely the correct moment (Wolf, 1976). In particular, reading musical notation is a visuospatial analysis task, in which information conveyed by spatial location and vertical separation of adjacent notes, is directly related to musical pitch interval, thus differing from the feature extraction task of reading printed alphabetic text (Sergent et al., 1992). Highly skilled sight readers are able to transform the array of visual symbols into the motor actions of musical performance at remarkable speed, an ability that is only acquired by practice (Kopiez et al., 2006). The nonmusical visuospatial skill of 3DMR is a high-level cognitive task that involves covert simulation of motor rotation requiring both maintenance and manipulation of the mental images. It is possible that faster 3DMR RT by musicians might be explained by better sensorimotor skills developed by years of daily instrument practice. However, this is not supported by RT data for either $0^{\circ}$ angle trials in $3 \mathrm{DMR}$ or the $2 \mathrm{D}$ shape-matching control tasks requiring only identity recognition, and which showed no difference between musicians and nonmusicians. The neurofunctional findings, therefore, suggest that Broca's area subserves the sequential mental manipulation components of 3DMR that are enhanced in orchestral musicians.

The behavioral findings that we report relating to slope of 3DMR RT function in musicians are comparable with previously reported RT slope functions of subjects who have undergone training, either on the 3DMR task itself (Kaushall and Parsons, 1981) or on related tasks for transfer to 3DMR (Wallace and Hofelich, 1992; Wiedenbauer et al., 2007), which show that training-enhanced performance is reflected in reduction of the slope of the RT function. Indeed, Kaushall and Parsons (1981) have shown previously that the slope of the RT function reduces to zero after sufficient numbers of 3DMR trials have been attempted (typically 864). However, musicians exhibited a flat slope RT function within the first five epochs of 3DMR and there was no evidence of a practice effect (in either musicians or nonmusicians) during this experiment. Although the limiting rate criterion (i.e., the fastest rate at which mental rotation could be performed), originally proposed by Shepard and Metzler (1988) was $15.6 \mathrm{~ms} /{ }^{\circ}$, there is now a wealth of evidence to support Cohen and Kubovy's (1993) proposal of $1 \mathrm{~ms} /{ }^{\circ}$ as a limiting value, which reflects the process of mental rotation. The gradient and intercept of the musicians' slope, $1.57 \mathrm{~ms} /{ }^{\circ}$ and $1403 \mathrm{~ms}$, can be taken to indicate that musicians were using a mental rotation strategy and, therefore, already possessed the neural circuitry to support this enhanced level of performance. Our finding that RT is negatively associated with number of years of orchestral playing may be the result of selection, if the less spatially skilled, younger musicians withdrew from the orchestra early in their performing career, which would also highlight the importance of visuospatial skills for professional orchestral musicians. An alternative interpretation of the negative correlation between RT and years of orchestral playing is that orchestral playing confers benefit to the performance of 3DMR and supports the notion that maintaining musical and sight-reading skills throughout adulthood may confer benefits on the more complex visuospatial tasks. Indeed, this interpretation is corroborated by the age-related improvement in performance observed in only musicians.

Both musicians and nonmusicians activated premotor, parietal, and visual association areas which are well known to sub- serve 3DMR (Cohen et al., 1996) and sight reading (Sergent et al., 1992). The only statistically significant between-group difference in activations, specifically within these commonly activated regions, was increased activation in the right angular gyrus (BA39) (Horwitz et al., 1998) of the posterior parietal cortex in musicians. BA39 has been reported to be activated during spatial localization (Rao et al., 2003), visuospatial attention (Nobre et al., 1997), coordinate judgment, and spatial memory (Chambers et al., 2004). Neuroanatomical and neurophysiological studies suggest a functional coupling between parietal and motor cortices when transforming perception into action (Rizzolatti et al., 1998). Indeed, connectivity is important because the functions that Broca's area supports will be influenced by its functional networks. Our finding of concomitant increased activations in Broca's area and the right angular gyrus may suggest a functional network supporting the spatial processing required for enhanced performance of 3DMR.

Although activations in pregenual ACC (areas BA24/32) were not predicted, this region is known to be activated during implementation of selected actions and particularly those with rapid responses (Bench et al., 1993; Naito et al., 2000). Increased activation within the left hemisphere in both Broca's area and pregenual ACC is consistent with the literature implicating ACC in attentionally demanding tasks (MacDonald et al., 2000), including music performance (Parsons et al., 2005), which suggests that ACC mediates cognitive control within the same hemisphere as the brain areas supporting the task being executed (Stephan et al., 2003). Furthermore, it has been suggested that lateral prefrontal cortex computes on-line information necessary for the choice of appropriate response whereas ACC facilitates implementation of the selected action (Paus, 2001) and, specifically, by monitoring conflicting movements rather than conflicting response choice (Cavina-Pratesi et al., 2006). These functions attributed to ACC are of particular importance for musical performance at a professional level.

In conclusion, we have shown that organization of the covert motor sequencing necessary for enhanced performance of 3DMR by musicians shares a neural substrate with the visuospatial sightreading skills of musical performance (Sergent et al., 1992; Parsons, 2001). Specifically in musicians, enhanced 3DMR performance was associated with increased BOLD signal within Broca's area, part of the sight-reading neural network (Sergent et al., 1992), which was negatively correlated with 3DMR RT and which we interpret to reflect exploitation of the neuroplastic adaptation of Broca's area in a larger group of musicians from the same orchestra that we observed in a previous investigation (Sluming et al., 2002). Orchestral musicians and controls, matched for handedness, age, and verbal intelligence, demonstrated a neural and behavioral dissociation on verbal and visuospatial tasks in that there were no differences in either behavioral data or Broca's area activation during performance of the verbal task, but there were significant differences, both behaviorally and neurofunctionally, during the visuospatial task performance. Our findings extend the current and growing literature reporting the transferable benefits of music performance training to nonmusical cognitive abilities in children and adolescents (Costa-Giomi, 2004; Schellenberg, 2004, 2005, 2006; Schlaug et al., 2005). Indeed, these findings have important implications, by providing additional objective evidence to support the suggestion that the development and maintenance of musical performance abilities confers benefit to nonmusical cognitive domains. 


\section{References}

Amunts K, Schleicher A, Burgel U, Mohlberg H, Uylings H, Zilles K (1999) Broca's region revisited: cytoarchitecture and intersubject variability. J Comp Neurol 412:319-341.

Baddeley A, Emslie H, Smith I (1992) The speed and capacity of language processing test. Bury St. Edmonds, UK: Thames Valley Test Company.

Barnes J, Howard RJ, Senior C, Brammer M, Bullmore ET, Simmons A, Woodruff P, David AS (2000) Cortical activity during rotational and linear transformations. Neuropsychologia 38:1148-1156.

Bench CJ, Frith CD, Grasby PM, Friston KJ, Paulesu E, Frackowiak RSJ, Dolan RJ (1993) Investigations of the functional anatomy of attention using the stroop test. Neuropsychologia 31:907.

Bengtsson SL, Ullen F (2006) Dissociation between melodic and rhythmic processing during piano performance from musical scores. NeuroImage 30:272-284

Binkofski F, Buccino G (2004) Motor functions of the Broca's region. Brain Lang 89:362-369.

Binkofski F, Amunts K, Stephan K, Posse S, Schormann T, Freund HJ, Zilles K, Seitz R (2000) Broca's region subserves imagery of motion: a combined cytoarchitectonic and fMRI study. Hum Brain Mapp 11:273-285.

Bonda E, Petrides M, Frey S, Evans A (1995) Neural correlates of mental transformations of the body-in-space. Proc Natl Acad Sci USA 92:11180-11184.

Carpentier A, Pugh KR, Westerveld M, Studholme C, Skrinjar O, Thompson J, Spencer D, Constable R (2001) Functional MRI of language processing: dependence on input modality and temporal lobe epilepsy. Epilepsia 42:1241-1254.

Cavina-Pratesi C, Valyear KF, Culham JC, Kohler S, Obhi SS, Marzi CA, Goodale MA (2006) Dissociating arbitrary stimulus-response mapping from movement planning during preparatory period: evidence from event-related functional magnetic resonance imaging. J Neurosci 26:2704-2713.

Chambers C, Payne J, Stokes M, Mattingley J (2004) Fast and slow parietal pathways mediate spatial attention. Nat Neurosci 7:217-218.

Chee MW, Hon NH, Caplan D, Lee HL, Goh J (2002) Frequency of concrete words modulates prefrontal activation during semantic judgments. NeuroImage 16:259-268.

Cohen D, Kubovy M (1993) Mental rotation, mental representation, and flat slopes. Cognit Psychol 25:351-382.

Cohen MS, Kosslyn SM, Breiter HC, DiGirolamo GJ, Thompson WL, Anderson AK, Bookheimer SY, Rosen BR, Belliveau JW (1996) Changes in cortical activity during mental rotation: a mapping study using functional MRI. Brain 119:89-100.

Collaer ML, Nelson JD (2002) Large visuospatial sex difference in line judgment: possible role of attentional factors. Brain Cogn 49:1-12.

Costa-Giomi E (2004) Effects of three years of piano instruction on children's academic achievement, school performance and self-esteem. Psychol Music 32:139-152.

Dror I, Kosslyn SM (1994) Mental imagery and aging. Psychol Aging 9:90-102.

Dror I, Schmitz-Williams IC, Smith W (2005) Older adults use mental representations that reduce cognitive load: mental rotation utilizes holistic representations and processing. Exp Aging Res 31:409-420.

Eickhoff SB, Heim S, Zilles K, Amunts K (2006) Testing anatomically specified hypotheses in functional imaging using cytoarchitectonic maps. NeuroImage 32:570-582.

Elbert T, Pantev C, Wienbruch C, Rockstroh B, Taub E (1995) Increased cortical representation of the fingers of the left hand in string players. Science 270:305-308.

Ericsson K, Krampe R, Tesch-Romer C (1993) The role of deliberate practice in the acquisition of musical skills. Psychol Rev 100:363-406.

Furneaux S, Land M (1999) The effects of skill on the hand-eye span during musical sght-reading. Proc R Soc Lond B Biol Sci 266:2435-2440.

Horwitz B, Rumsey JM, Donohue BC (1998) Functional connectivity of the angular gyrus in normal reading and dyslexia. Proc Natl Acad Sci USA 95:8939-8944.

Just M, Carpenter P (1985) Cognitive coordinate systems: accounts of mental rotation and individual differences in spatial ability. Psychol Rev 92:137-172.
Kaushall P, Parsons L (1981) Optical information and practice in the discrimination of 3D mirror reflected objects. Perception 10:545-562.

Koechlin E, Jubault T (2006) Broca's area and the hierarchical organization of human behavior. Neuron 50:963-974.

Kopiez R, Weihs C, Ligges U, Lee J (2006) Classification of high and low achievers in a music sight-reading task. Psychol Music 36:5-26.

Kosslyn SM, Brunn J, Cave KR, Wallach RW (1984) Individual differences in mental imagery ability: a computational analysis. Cognition 18:195.

MacDonald AW, Cohen JD, Stenger VA, Carter CS (2000) Dissociating the role of the dorsolateral prefrontal and anterior cingulate cortex in cognitive control. Science 288:1835-1838.

McPherson GE, Bailey M, Sinclair KE (1997) Path analysis of a theoretical model to describe the relationship among five types of musical performance. J Res Music Educ 45:103-129.

Mechelli A, Crinion JT, Long S, Friston KJ, Ralph MAL, Patterson K, McClelland JL, Price CJ (2005) Dissociating reading processes on the basis of neuronal interactions. J Cogn Neurosci 17:1753-1765.

Naito E, Kinomura S, Geyer S, Kawashima R, Roland PE, Zilles K (2000) Fast reaction to different sensory modalities activates common fields in the motor areas, but the anterior cingulate cortex is involved in the speed of reaction. J Neurophysiol 83:1701-1709.

Ng VW, Bullmore ET, de Zubicaray GI, Cooper A, Suckling J, Williams SC (2001) Identifying rate-limiting nodes in large-scale cortical networks for visuospatial processing: an illustration using fMRI. J Cogn Neurosci 13:537-545.

Nobre A, Sebestyen G, Gitelman D, Mesulam M, Frackowiak R, Frith C (1997) Functional localization of the system for visuospatial attention using positron emission tomography. Brain 120:515-533.

Parsons LM (2001) Exploring the functional neuroanatomy of music performance, perception and comprehension. In: The biological foundations of music (Zatorre R, Peretz I, eds), pp 211-231. New York: New York Academy of Sciences.

Parsons LM, Fox P, Downs J, Glass T, Hirsch T, Martin C, Jerabek P, Lancaster J (1995) Use of implicit motor imagery for visual shape discrimination as revealed by PET. Nature 375:54-58.

Parsons LM, Sergent J, Hodges D, Fox P (2005) The brain basis of piano performance. Neuropsychologia 43:199-215.

Paus T (2001) Primate anterior cingulate cortex: where motor control, drive and cognition meet. Nat Rev Neurosci 2:417-424.

Rao H, Zhou T, Zhou Y, Fan S, Chen L (2003) Spatiotemporal activation of the two visual pathways in form discrimination and spatial location. Hum Brain Mapp 18:79-89.

Rizzolatti G, Luppino G, Matelli M (1998) The organization of the cortical motor system: new concepts. Electroencephalogr Clin Neurophysiol 106:283-296.

Saxton J, Ratcliff G, Dodge H, Pandav R, Baddeley A, Ganguli M (2001) Speed and capacity of language processing test: normative data from an older American community-dwelling sample. Appl Neuropsychol 8:193-203.

Schellenberg EG (2004) Music lessons enhance IQ. Psychol Sci 15:511-514

Schellenberg EG (2005) Music and cognitive abilities. Curr Dir Psychol Sci 14:317-320.

Schellenberg EG (2006) Long-term positive associations between music lessons and IQ. J Educ Psychol 98:457-468.

Schlaug G, Jancke L, Huang Y, Staiger JF, Steinmetz H (1995) Increased corpus callosum size in musicians. Neuropsychologia 33:1047-1055.

Schlaug G, Norton A, Overy K, Winner E (2005) Effects of music training on the child's brain and cognitive development. Ann NY Acad Sci 1060:219-230.

Schneider P, Sluming V, Roberts N, Scherg M, Goebel R, Specht HJ, Dosch HG, Bleek S, Stippich C, Rupp A (2005) Structural and functional asymmetry of lateral Heschl's gyrus reflects pitch perception preference. Nat Neurosci 8:1241-1247.

Sergent J (1993) Mapping the musician brain. Hum Brain Mapp 1:20-38.

Sergent J, Zuck E, Terriah S, MacDonald B (1992) Distributed neural network underlying musical sight-reading and keyboard performance. Science 257:106-109.

Shepard R, Metzler J (1971) Mental rotation of three-dimensional objects. Science 171:701-703.

Shepard RN, Cooper LA (1982) Mental images and their transformation. Cambridge, MA: MIT. 
Shepard S, Metzler D (1988) Mental rotation: effects of dimensionality of objects and type of task. J Exp Psychol Hum Percept Perform 14:3-11.

Sluming V, Barrick T, Howard M, Cezayirli E, Mayes A, Roberts N (2002) Voxel-based morphometry reveals increased gray matter density in Broca's area in male symphony orchestra musicians. NeuroImage 17:1613-1622.

Sowell E, Thompson P, Holmes C, Jernigan T, Toga A (1999) In vivo evidence for post adolescent brain maturation in the frontal and striatal regions. Nat Neurosci 2:859-861.

Stephan K, Fink G, Passingham R, Silbersweig D, Ceballos-Baumann A, Frith C, Frackowiak R (1995) Functional anatomy of the mental representation of upper extremity movements in healthy subjects. J Neurophysiol 73:373-385.
Stephan KE, Marshall JC, Friston KJ, Rowe JB, Ritzl A, Zilles K, Fink GR (2003) Lateralized cognitive processes and lateralized task control in the human brain. Science 301:384-386.

Sterr A, Muller M, Elbert T (1998) Changed perceptions in Braille readers. Nature 391:134-135.

Wallace B, Hofelich B (1992) Process generalisation and the prediction of performance on mental imagery tasks. Mem Cognit 20:695-704.

Waters A, Underwood G, Findlay J (1997) Studying expertise in music reading: use of a pattern-matching paradigm. Percept Psychophys 59:477-488.

Wiedenbauer G, Schmid J, Jansen-Osmann P (2007) Manual training of mental rotation. Eur J Cogn Psychol 19:17-36.

Wolf T (1976) A cognitive model of musical sight-reading. J Psycholinguist Res 5:143-171. 